\title{
Neutrophil to Lymphocite Ratio Predicts Overall Survival in Newly Diagnosed Hodgkin Lymphoma Patients-Single Centre Experience
}

\author{
Jelena Hajder ${ }^{1}$, Natasa Stanisavljević ${ }^{1}$, Dragomir Marisavljevićc, ${ }^{1,2}$ Olivera Marković, ${ }^{1,2}$, \\ Radmila Zivković ${ }^{1}$ and Vladimir Jurišić ${ }^{3}$ \\ ${ }^{1}$ Medical Center "Bezanijska kosa", Clinic for hematology and oncology, Serbia \\ ${ }^{2}$ Faculty of Medical Sciences, University of Belgrade, Serbia \\ ${ }^{3}$ Faculty of Medical Sciences, Univeristy of Kragujevac, Serbia
}

\begin{abstract}
Introduction: Neutrophil to lymphocyte ratio (NLR) has been identified as an independent prognostic factor for overall survival (OS) in various types of cancer.

Aim: The aim of this study was to establish prognostic significance of NLR in patients with newly diagnosed HL.

Methods: This retrospective analysis included 83 newly diagnosed patients with HL followed up from 2001-2015. ROC curve analysis was used to determine the optimal cut-off values for overall survival. Univariate and multivariate analysis was performed using the Cox proportional hazards regression to identify significant independent prognostic factors.

Results: The median follow-up after diagnosis was 92 months for the entire cohort (range 9-180 months) and 25.5 months for censored patients (range 1-81 months). Complete remission (CR) was achieved in 63 (79.7\%) patients. An ROC curve of the NLR considering overall survival was generated showing AUC $0.655 \pm 0.064(95 \% \mathrm{Cl}, 0.536-0.724)$ with cutoff value of 4.3 ( $66 \%$ sensitivity and $59 \%$ specificity). Median OS for patients with NLR $\leq 4.3$ was 61 months vs 50 months for patients with NLR $\geq 4.3,(p<0.018)$. Multivariate analysis showed that independent prognostic variables for OS were high IPS $(\geq 4)(p=0.013)$, NLR ratio $\geq 4.3(p=0.007)$ and age $\geq 45$, $(p=0.001)$. Patients in (un)favorable stage with NLR $\geq$ 4.3 in comparison to patients with NLR $\leq 4.3$ had poorer survival in early unfavorable and advanced stage in contrast to advanced disease according to GHSG and EORTC staging system.

Conclusion: High NLR $(\geq 4.3)$ is independent adverse prognostic factor for overall survival and should be considered as useful biomarker for precise prediction of clinical outcomes in early stage $\mathrm{HL}$.
\end{abstract}

\section{Keywords}

Hodgkin lymphoma, Neutrophil, Lymphocyte, Neutrophil/Lymphocyte ratio, Prognosis, Early stage disease

\section{List of Abbreviation}

ABVD: Doxorubicin, Bleomycin, Vinblastine, Dacarbasine f; ABVD/MOPP: Hybrid Mechlorthamine, Vincristine, Procarbasine, and Prednisone; ALC: Absolute Lymphocyte Count; ANC: Absolute Neutrophil Count; BEACOPP: Bleomycin, Etoposide, Doxorubicin, Cyclophosphamide, Vincristine, Procarbasine, Prednisolone; CBC: Complete Blood Count; CR: Complete Remission; DLBCL: Diffuse Large B Cell Lymphoma; EORTC: European Organization for the Research and Treatment in Cancer; GHSG: German Hodgkin Study Group; HBV: Hepatitis B Virus; HCV: Hepatitis C Virus; HIV: Human Immunodeficiency Viruses; HL: Hodgkin Lymphoma; IPS: International Prognostic Score; LDH: Lactate Dehydrogenase; MTB: Mycobacterium Tuberculosis; NLR: Neutrophil to Lymphocyte Ratio; NS/MC: Nodular Sclerosis/Mixed Cellularity; OS: Overall Survival; PFS: Progression Free Survival; ROC: Receiver Operating Curve; WBC: Leukocyte,White Blood Cells

\section{Introduction}

Hodgkin lymphoma $(\mathrm{HL})$ is a B-cell derived lymphoid malignancy mainly affecting younger adults in their third and fourth decade of life. HL has become a curable malignancy with more than $90 \%$ of patients alive and $80 \%$ considered cured after a minimal follow-up of 6 years [1]. However, current therapies for this disease can have significant shortterm and long -term toxicity. Thus, treatment focuses on tailoring therapy according to prognostic schemes that imply
*Corresponding author: Jelena Hajder, Medical Center "Bezanijska kosa", Clinic for hematology and oncology, Belgrade, Serbia Accepted: August 16, 2021

Published online: August 18, 2021

Citation: Hajder J, Stanisavljević N, Marisavljević D, et al. (2021) Neutrophil to Lymphocite Ratio Predicts Overall Survival in Newly Diagnosed Hodgkin Lymphoma Patients-Single Centre Experience. Clin Hematol Res 4(1):47-54 
Citation: Hajder J, Stanisavljević N, Marisavljević D, et al. (2021) Neutrophil to Lymphocite Ratio Predicts Overall Survival in Newly Diagnosed Hodgkin Lymphoma Patients-Single Centre Experience. Clin Hematol Res 4(1):47-54

clinical risk factors to predict the risk for disease progression, relapse and death of patients with $\mathrm{HL}$. The International Prognostic Score (IPS) uses seven prognostic factors to predict clinical outcomes in newly diagnosed $\mathrm{HL}$ patients [2]. Adverse prognostic factors in classic $\mathrm{HL}$ are age $>45$ years, stage IV disease, hemoglobin $<10 \mathrm{~g} / \mathrm{l}$, lymphocyte count $<0.6$ $\times 10^{9} / \mathrm{L}$, male sex, albumin $<40 \mathrm{~g} / \mathrm{L}$, white blood count $>15$ $\times 10^{9} / \mathrm{l}$. However, the IPS does not offer risk stratification for $\mathrm{HL}$ patients with limited disease (i.e., stages I and IIA, without constitutional symptoms and no bulky disease $[\geq 10 \mathrm{~cm}$ in diameter]).

Inflammation has been identified to be a critical component of tumor progression, highlighting the role of the microenvironment, which is largely orchestrated by inflammatory cells as an indispensable participant in the neoplastic process, fostering proliferation, survival and migration [3]. For different solid tumors, as well as lymphomas, inflammation parameters, including leukocytes, neutrophils, lymphocytes and C-reactive protein have been associated with higher mortality rates [4-6]. Neutrophil to lymphocyte ratio (NLR) may reflect and clarify the immune response in systemic inflammatory response. NLR has been identified as an independent prognostic factor for overall survival (OS) and progression free survival (PFS) in various types of cancer, including colorectal cancer [7], pancreatic cancer [8], renal cell,sarcoma [9] carcinoma [10], and in diffuse large B cell lymphoma (DLBCL) $[6,11]$. We studied whether NLR at diagnosis can predict treatment response and overall survival in patients with classic $\mathrm{HL}$. We then correlated the results of this analysis with other well known risk stratification systems.

\section{Materials and Methods}

This retrospective study included 83 consecutive patients diagnosed with $\mathrm{HL}$ at Medical Center "Bezanijska kosa", Belgrade, Serbia from 2001-2015. All patients met the following criteria: Pathohistologicaly confirmed $\mathrm{HL}$ without previous treatment, no previous history of malignancy, negativity for HIV, HBV, HCV or TBC infection with availability of laboratory and follow up data. Clinical data were retrieved from medical records. Treatment options and response criteria were based on standard guidelines. The ANC and ALC were obtained from the routine complete blood count (CBC) with 4-part differential counts (lymphocytes, monocytes, eosinophils, and neutrophils) using $A B X$ Pentra DX-DF 120-HORIBA and NLR was calculated for each patient at diagnosis. The study was conducted according to the ethical guidelines following the Declaration of Helsinki. The institutional review committee approved our study protocol thereby following local biomedical research regulations. Statistical analysis Categorical variables were compared by using $\chi^{2}$ test (2-sided Pearson or linear-By-linear association). Continuous variables were compared with the Mann-Whitney test.

The determination of optimal cut-off value for the ANC and ALC ratio in predicting overall survival was performed using the Receiver Operating Curve (ROC) method with determination of sensitivity and specificity of all cut-off values. OS was defined as time between the first day of diagnosis and the date of death from any cause or the last follow-up. Spearman's rank correlation coefficient was used to describe the correlation between quantitative variables. The KaplanMeier method was used to estimate overall survival (OS). The log-rank test was used to compare the survival distributions. A multivariate analysis (Cox regression method) was performed to examine the effect of presumed prognostic factors on the overall survival. All statistical analyses were performed using the IBM SPSS version 22.0 statistical software program (SPSS, Chicago, IL). The results were considered to be statistically significant when the $p$ value was less than 0.05

\section{Results}

\section{Patient characteristics}

Patient age ranged from 18 to 80 years (median, 42 years). There were $44(53 \%)$ male and $39(47 \%)$ female patients. Nodular sclerosis/mixed cellularity (NS/MC) was the most common histological subtype, noted in 39 (47\%) patients. Nodular sclerosis (NS) was the second common subtype, noted in $27(32.5 \%)$ patients, followed by seven (8.4\%) cases of the mixed cellularity, six (7.3\%) cases of lymphocyte rich and four (4.8\%) cases of the lymphocyte depletion. According to Ann Arbor staging system of $\mathrm{HL}, 3(3.6 \%)$ patients were staged as stage I, 42 (50.6\%) were stage II, 20 (24.1\%) were stage III, and $18(21.7 \%)$ were stage IV. German Hodgkin study Group (GSHG) early-stage favorable HL was noted in 16 (19.3\%), early-stage (un)favorable $\mathrm{HL}$ in 28 (33.7\%) and advancedstage $\mathrm{HL}$ in 39 (47\%) patients. European Organization for the Research and Treatment in Cancer (EORTC) early-stage favorable HL was noted in 13 (15.7\%) patients and early-stage (un)favorable $\mathrm{HL}$ in 30 (36.1\%) patients, whilst most patients (40 or $48.2 \%$ ) were in advanced-stage $\mathrm{HL}$ on presentation. Extra -nodal lymphoma was present in 12 (14.5\%) patients. Bulky disease (the largest lymph node $>10 \mathrm{~cm}$ ) was noted in 26 (31.3\%) patients. B symptoms were present in 43 (53.8\%) patients. IPS $\geq 4$ was noted in $18(21.7 \%)$ patients. Increased serum levels of lactate dehydrogenase (LDH) were found in 25 (30.1\%) patients. Hemoglobin lower than $105 \mathrm{~g} / \mathrm{L}$ was found in $21(25.3 \%)$ patients and leukocytosis (WBC > $15 \times$ $\left.10^{9}\right)$ in $11(13.3 \%)$ patients.

Patients were treated with standard chemotherapy and/ or radiotherapy according to current international guidelines considering stage of the disease and risk factors. A majority of patients $47(51 \%)$ received chemotherapy only and 44 (47.8\%) patients were treated with chemo radiotherapy. Treatment regiments included doxorubicin, bleomycin, vinblastine, and dacarbasine (ABVD) for 75 (81.5\%) patients, mechlorthamine, vincristine, procarbasine, and prednisone (MOPP) for 1 (1.1\%), ABVD/MOPP hybrid 9 (9.98\%), bleomycin, etoposide, doxorubicin, cyclophosphamide, vincristine, procarbasine, and prednisolone (BEACOPP) for 7 (7.6\%) patients. The median follow-up after diagnosis was 92 months for the entire cohort (range 9-180 months) and 25.5 months for censored patients (range 1-81 months). Complete remission (CR) was achieved in $63(79.7 \%)$ patients (Table 1$)$.

\section{Determining the cut-off value of the NLR}

An ROC curve of the NLR (ANC/ALC ratio) according to 
Citation: Hajder J, Stanisavljević N, Marisavljević D, et al. (2021) Neutrophil to Lymphocite Ratio Predicts Overall Survival in Newly Diagnosed Hodgkin Lymphoma Patients-Single Centre Experience. Clin Hematol Res 4(1):47-54

Table 1: Patients characteristics by NLR.

\begin{tabular}{|c|c|c|c|c|c|c|}
\hline & & \multicolumn{2}{|c|}{$N L R \leq 4.3$} & \multicolumn{2}{|c|}{ NLR $>4.3$} & \multirow{2}{*}{ significance } \\
\hline & & $n$ & $\%$ & $n$ & $\%$ & \\
\hline \multirow{2}{*}{ Gender } & male & 23 & 59.0 & 21 & 47.7 & \multirow{2}{*}{ ns } \\
\hline & female & 16 & 41.0 & 23 & 52.3 & \\
\hline \multirow{2}{*}{ Age } & $<45$ years & 20 & 51.3 & 26 & 59.1 & \multirow{2}{*}{ ns } \\
\hline & $\geq 45$ years & 19 & 48.7 & 18 & 40.9 & \\
\hline \multirow{2}{*}{ Hemoglobin level } & $>10.5 \mathrm{~g} / \mathrm{dl}$ & 29 & 74.4 & 33 & 75.0 & \multirow{2}{*}{ ns } \\
\hline & $<10.5 \mathrm{~g} / \mathrm{dl}$ & 10 & 25.6 & 11 & 25.0 & \\
\hline \multirow{2}{*}{ White blood cell count } & $<15,000 / \mu l$ & 36 & 92.3 & 36 & 81.8 & \multirow{2}{*}{ ns } \\
\hline & $\geq 15,000 / \mu \mathrm{l}$ & 3 & 7.7 & 8 & 18.2 & \\
\hline \multirow{2}{*}{ Lymphocyte count } & $<600 / \mu$ or $<8 \%$ & 3 & 7.7 & 11 & 25.0 & \multirow{2}{*}{$0.036^{*}$} \\
\hline & $\geq 600 / \mu$ or $\geq 8 \%$ ne & 36 & 92.3 & 33 & 75.0 & \\
\hline \multirow{2}{*}{ Elevated LDH } & no & 27 & 69.2 & 31 & 70.5 & \multirow{2}{*}{ ns } \\
\hline & yes & 12 & 30.8 & 13 & 29.5 & \\
\hline \multirow[t]{2}{*}{ B symptoms } & no & 20 & 52.6 & 17 & 40.5 & \multirow{2}{*}{ ns } \\
\hline & yes & 18 & 47.4 & 25 & 59.5 & \\
\hline \multirow{5}{*}{$\begin{array}{l}\mathrm{PH} \\
\text { subtype }\end{array}$} & MC & 4 & 10.3 & 3 & 6.8 & \multirow{5}{*}{ ns } \\
\hline & NS & 17 & 43.6 & 10 & 22.7 & \\
\hline & LD & 3 & 7.7 & 1 & 2.3 & \\
\hline & LP & 3 & 7.7 & 3 & 6.8 & \\
\hline & NS/MC & 12 & 30.8 & 27 & 61.4 & \\
\hline \multirow{4}{*}{$\begin{array}{l}\text { Clinical } \\
\text { stage }\end{array}$} & 1 & 1 & 2.6 & 2 & 4.5 & \multirow{4}{*}{ ns } \\
\hline & II & 23 & 59.0 & 19 & 43.2 & \\
\hline & III & 9 & 23.1 & 11 & 25.0 & \\
\hline & IV & 6 & 15.4 & 12 & 27.3 & \\
\hline \multirow{5}{*}{ ECOG } & 0 & 23 & 60.5 & 19 & 45.2 & \multirow{5}{*}{ ns } \\
\hline & 1 & 9 & 23.7 & 12 & 28.6 & \\
\hline & 2 & 3 & 7.9 & 5 & 11.9 & \\
\hline & 3 & 3 & 7.9 & 4 & 9.5 & \\
\hline & 4 & 0 & 0.0 & 2 & 4.8 & \\
\hline \multirow{2}{*}{ IPS } & $<4$ & 32 & 82.1 & 33 & 75.0 & \\
\hline & $\geq 4$ & 7 & 17.9 & 11 & 25.0 & ns \\
\hline Bulky & no & 29 & 74.4 & 28 & 63.6 & \\
\hline disease & yes & 10 & 25.6 & 16 & 36.4 & ns \\
\hline Extranodal & no & 34 & 87.2 & 37 & 84.1 & \\
\hline disease & yes & 5 & 12.8 & 7 & 15.9 & ns \\
\hline & early favorable & 10 & 25.6 & 6 & 13.6 & \\
\hline GHSG & early unfavorable & 13 & 33.3 & 15 & 34.1 & ns \\
\hline & advanced & 16 & 41.0 & 23 & 52.3 & \\
\hline & early favorable & 8 & 20.5 & 5 & 11.4 & \\
\hline EORTC & early unfavorable & 16 & 41.0 & 14 & 31.8 & ns \\
\hline & advanced & 15 & 38.5 & 25 & 56.8 & \\
\hline Resnonse to treatment $(\mathrm{N}=79)$ & $\mathrm{CR}$ & 30 & 85.7 & 33 & 75.0 & $n c$ \\
\hline Response to treatment $(\mathrm{N}=/ \mathrm{Y})$ & PR, SD, progression & 5 & 14.3 & 11 & 25.0 & ns \\
\hline
\end{tabular}


Citation: Hajder J, Stanisavljević N, Marisavljević D, et al. (2021) Neutrophil to Lymphocite Ratio Predicts Overall Survival in Newly Diagnosed Hodgkin Lymphoma Patients-Single Centre Experience. Clin Hematol Res 4(1):47-54

\begin{tabular}{|l|l|l|l|l|l|l|}
\hline \multirow{2}{*}{ Treatment outcome } & dead & 8 & 20.5 & 21 & 48.8 & $0.007^{* *}$ \\
\cline { 2 - 6 } & alive & 31 & 79.5 & 22 & 51.2 & 2.3 \\
\cline { 2 - 5 } & lost in follow up & 0 & 0.0 & 1 & \\
\hline
\end{tabular}

N: Number; NS: No Significance; CR: Complete Remission; PR: Partial Remission, SD: Stabile Disease

A

Survival Function

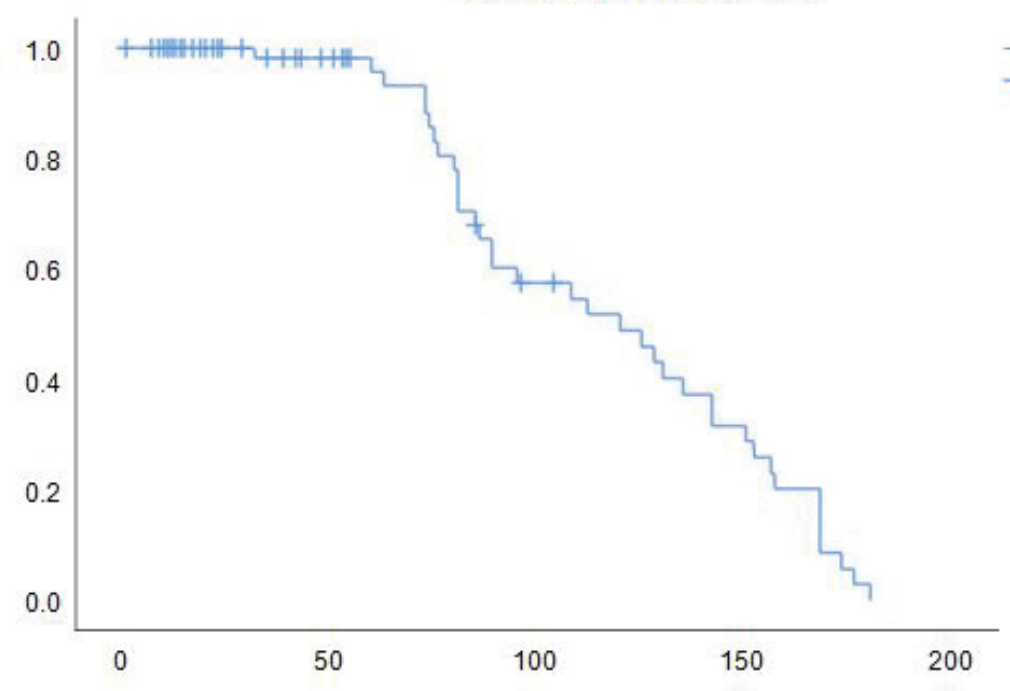

overall survival

B

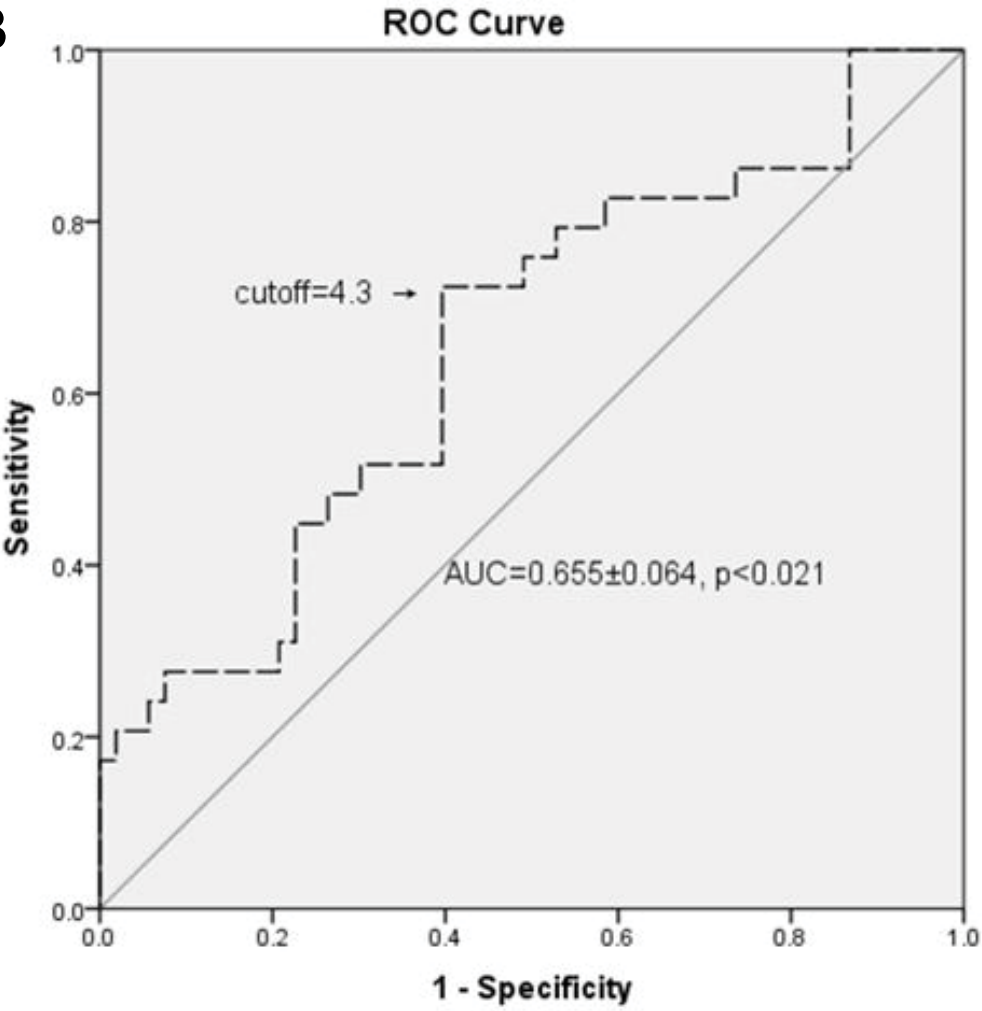

Figure 1: $(a, b)$ ROC curve of the NLR (ANC/ALC ratio) according to overall survival. 
overall survival was generated to determine the cut-off value. The area under the curve was recorded as $0.655 \pm 0.064$ (95\% $\mathrm{Cl}, 0.536-0.724$ ) (Figure 1). The NLR value of 4.3 corresponded to the maximum combined sensitivity and specificity on the ROC curve (66\% sensitivity and $59 \%$ specificity). Therefore, median OS for patients with NLR $\leq 43$ was 61 months and for subgroup with high NLR (NLR $\geq 4.3$ ) median OS was 50 months. The clinic pathological features of the patients with $\mathrm{NLR} \leq 4.3$ and those with an NLR $\geq 4.3$, are summarized in Table 1. There were 39 (47\%) with NLR $\leq 4.3$ and 44 (53\%) with NLR $\geq 4.3$.

\section{Prognostic significance of the NLR (ANC/ALC ratio)}

There was significant difference in OS between patients with NLR $\geq 4.3$ vs. patients with NLR $\leq 4.3$ ( $p<0.018$ ) (Figure 2). Univariate analysis revealed that OS significantly correlated with older age ( $>45$ years), lymphopenia $(<600 / \mu \mathrm{l}$ vs. $\geq 600 / \mu \mathrm{l}$ ), NLR ratio (continuous variable), NLR $\geq 4.3$, male sex, advanced clinical stage, poor risk according IPS, GHSG and EORTC staging, and lower than CR as treatment response (Table 2). Multivariate analysis showed that independent

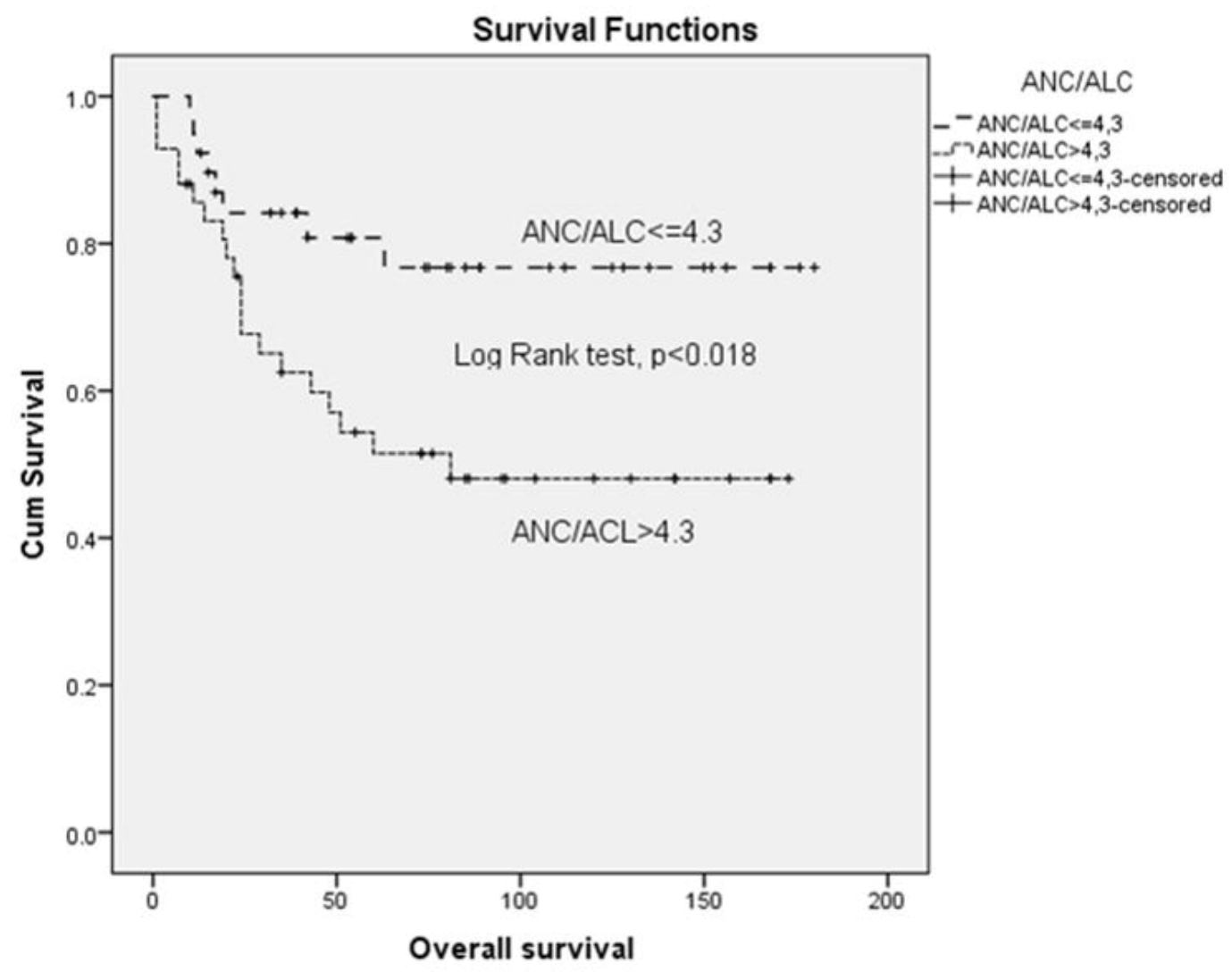

Figure 2: Comparison of OS of $H L$ patients with NLR $\geq 4.3$ and patients with NLR $\leq 4.3$.

Table 2: Univariate analysis for OS in patients with classic HL.

\begin{tabular}{|c|c|c|c|c|c|c|c|}
\hline \multirow{2}{*}{ Cox analysis } & & \multirow[b]{2}{*}{ B } & \multirow[b]{2}{*}{ SE } & \multirow[b]{2}{*}{$P$ value } & \multirow[b]{2}{*}{ HR } & \multicolumn{2}{|c|}{ 95.0\% Cl for $\operatorname{Exp}(\mathrm{B})$} \\
\hline & & & & & & Lower & Upper \\
\hline \multicolumn{8}{|l|}{ Univariate Analysis for OS } \\
\hline Sex & Fvs. M & 0.296 & 0.379 & 0.435 & 1.344 & 0.640 & 2.826 \\
\hline Age & $<45$ vs. $\geq 45$ & 1.607 & 0.438 & 0.000 & 4.985 & 2.115 & 11.753 \\
\hline $\begin{array}{l}\text { Hemoglobin } \\
(\mathrm{g} / \mathrm{L})\end{array}$ & $\begin{array}{l}\geq 105 \\
\leq 105\end{array}$ & 0.296 & 0.405 & 0.465 & 1.345 & 0.608 & 2.975 \\
\hline Elevated LDH, U/L & No vs yes & 0.281 & 0.395 & 0.476 & 1.325 & 0.611 & 2.871 \\
\hline Lymphocyte count $\left(\times 10^{9} / I\right)$ & $\geq 0.6$ vs. $<0.6$ & -1.140 & 0.421 & 0.007 & 0.320 & 0.140 & 0.730 \\
\hline NLR ratio & continuous & 0.089 & 0.032 & 0.005 & 1.093 & 1.027 & 1.163 \\
\hline NLR & $\leq 4.3$ vs. $\geq 4.3$ & 0.946 & 0.419 & 0.024 & 2.575 & 1.134 & 5.849 \\
\hline
\end{tabular}


Citation: Hajder J, Stanisavljević N, Marisavljević D, et al. (2021) Neutrophil to Lymphocite Ratio Predicts Overall Survival in Newly Diagnosed Hodgkin Lymphoma Patients-Single Centre Experience. Clin Hematol Res 4(1):47-54

\begin{tabular}{|l|l|l|l|l|l|l|l|}
\hline WBC $\left(\times 10^{9} / \mathrm{I}\right)$ & $\leq 15$ vs. $\geq 15$ & 0.518 & 0.461 & 0.261 & $\mathbf{1 . 6 7 9}$ & 0.680 & 4.148 \\
\hline B symptoms & $(-)$ vs $(+)$ & 0.144 & 0.397 & 0.717 & $\mathbf{1 . 1 5 5}$ & 0.530 & 2.515 \\
\hline Stage & continuous & 0.478 & 0.214 & $\mathbf{0 . 0 2 6}$ & $\mathbf{1 . 6 1 3}$ & 1.060 & 2.455 \\
\hline Stage & $1-3$ vs. 4 & 0.620 & 0.405 & 0.126 & $\mathbf{1 . 8 6 0}$ & 0.841 & 4.114 \\
\hline GHSG & continuous & 0.890 & 0.322 & $\mathbf{0 . 0 0 6}$ & $\mathbf{2 . 4 3 4}$ & 1.295 & 4.576 \\
\hline EORTC & continuous & 0.892 & 0.341 & $\mathbf{0 . 0 0 9}$ & $\mathbf{2 . 4 4 0}$ & 1.251 & 4.759 \\
\hline IPS & continuous & 0.448 & 0.135 & $\mathbf{0 . 0 0 1}$ & $\mathbf{1 . 5 6 5}$ & 1.202 & 2.039 \\
\hline IPS & $<4$ vs. $\geq 4$ & 1.346 & 0.384 & $\mathbf{0 . 0 0 0}$ & $\mathbf{3 . 8 4 2}$ & 1.811 & 8.152 \\
\hline Treatment outcome & Dead/alive & 3.406 & 0.518 & $\mathbf{0 . 0 0 0}$ & $\mathbf{3 0 . 1 5 3}$ & 10.925 & 83.226 \\
\hline
\end{tabular}

Table 3: Multivariate Analysis for OSin patients with classic HL.

\begin{tabular}{|l|l|l|l|l|l|l|l|}
\hline \multicolumn{2}{|l|}{ Variable } & B & SE & P value & HR & \multicolumn{3}{|l|}{$\mathbf{9 5 . 0 \% ~ C l ~ f o r ~ E x p ( B ) ~}$} \\
\hline IPS & $<4$ vs. $\geq 4^{*}$ & 1.049 & 0.423 & $\mathbf{0 . 0 1 3}$ & 2.854 & 1.245 & 6.543 \\
\hline NLR & $\leq 4.3$ vs. $>4.3$ & 1.181 & 0.441 & $\mathbf{0 . 0 0 7}$ & 3.257 & 1.371 & 7.739 \\
\hline Lymphocyte count & $\begin{array}{l}\geq 600 / \mu \text { l vs. }< \\
600 / \mu \mathrm{l}\end{array}$ & 0.852 & 0.465 & 0.067 & 0.427 & 0.172 & 1.060 \\
\hline Age & $<45$ vs. $\geq 45$ & 1.534 & 0.476 & $\mathbf{0 . 0 0 1}$ & 4.638 & 1.823 & 11.796 \\
\hline
\end{tabular}

A)

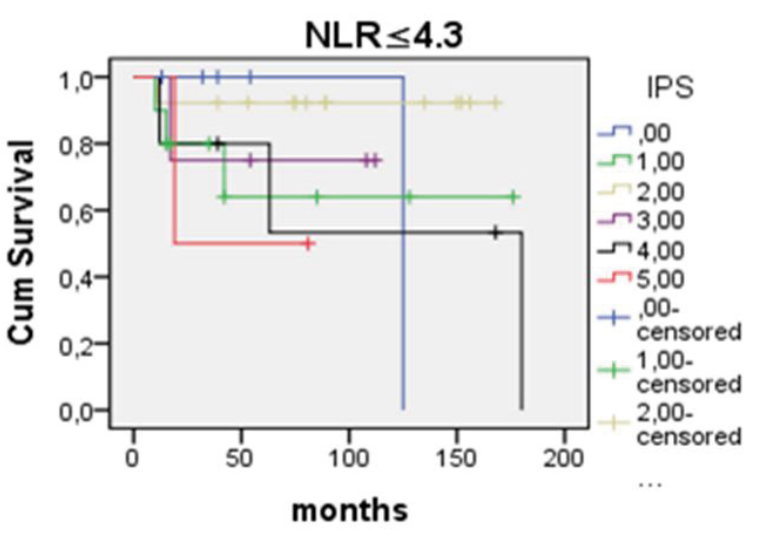

B) Survival Functions

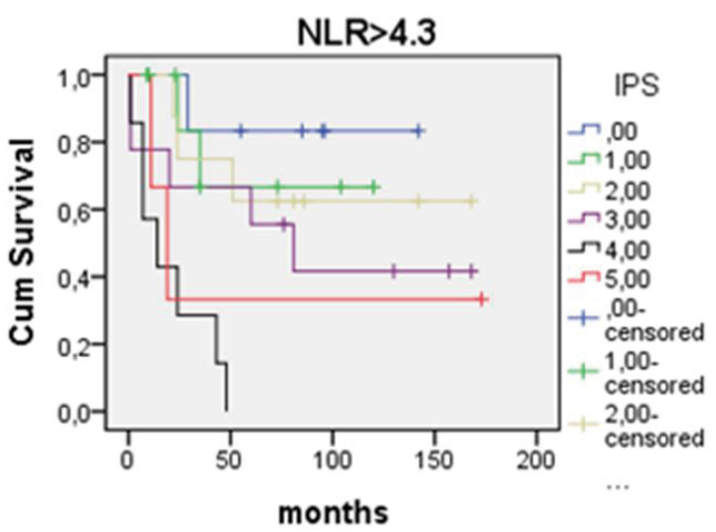

Figure 3: $(a, b)$ Comparison of OS of HL patients with NLR $\geq 4.3$ vs. NLR $\leq 4.3$ considering different IPS score.

prognostic variables for OS were high IPS $(\geq 4)(p=0.013)$, NLR $\geq 4.3(p=0.007)$ and age $\geq 45(p=0.001)$ (Table 3$)$. Patients with NLR $\leq 4.3$ in comparison to patients with NLR $\geq 4.3$, had longer survival considering any IPS (Figure 3 and Figure 4), but high NLR was particularly poor risk factor in patients with IPS 4 (log rank $=0.004$ ) (Figure 3 and Figure 4). Taking in account that IPS was generated for patients with advanced stage $\mathrm{HL}$, we future explored the role of NLR according to both, GSHG and EORTC stage of HL [2] (Figure 4 and Figure 5). Results clearly showed that patients with NLR $\geq 4.3$ in comparison to patients with NLR $\leq 4.3$ had poorer survival in early (un) favorable in comparison to advanced stage disease.

\section{Discussion}

This study has few important aspects. First we clearly showed that NLR can reliable identify patients who will have a shorter survival, alone or in respect to other well known staging systems. Second, most of the studies on the significance of NLR in HL reported so far are multi centric studies [12-14]. Our study is a single centre study with limitations of retrospective nature of the study designed and relatively small number of patients, but nevertheless informative enough and conclusive. In addition, it seems that NLR improve estimation of prognosis in early (un) favorable stage of the disease according to GHSG and EORTIC staging systems.

Although $\mathrm{HL}$ is considered a highly curable disease, $20 \%$ of patients cannot be cured with standard first-line chemotherapy and have a dismal outcome. Since current clinical parameters do not allow precise risk stratification 
A)

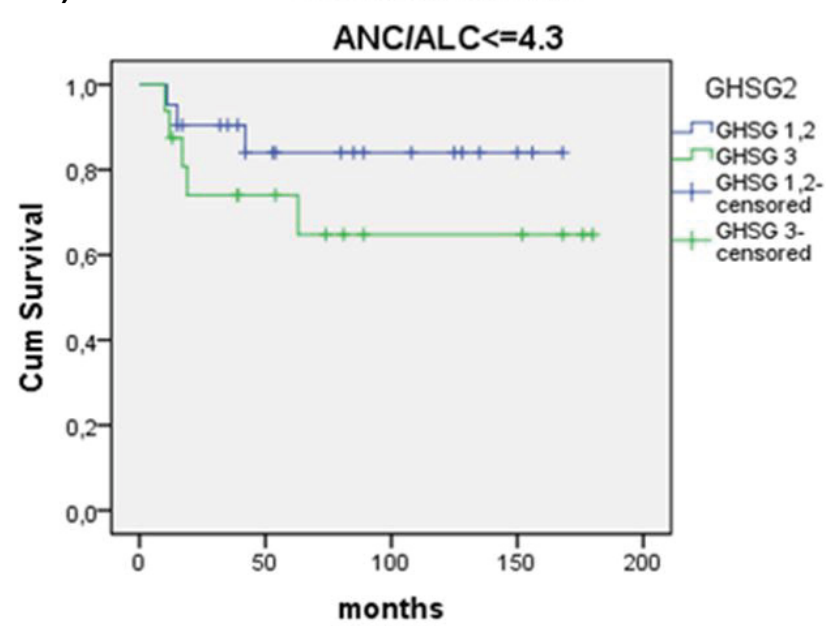

B)

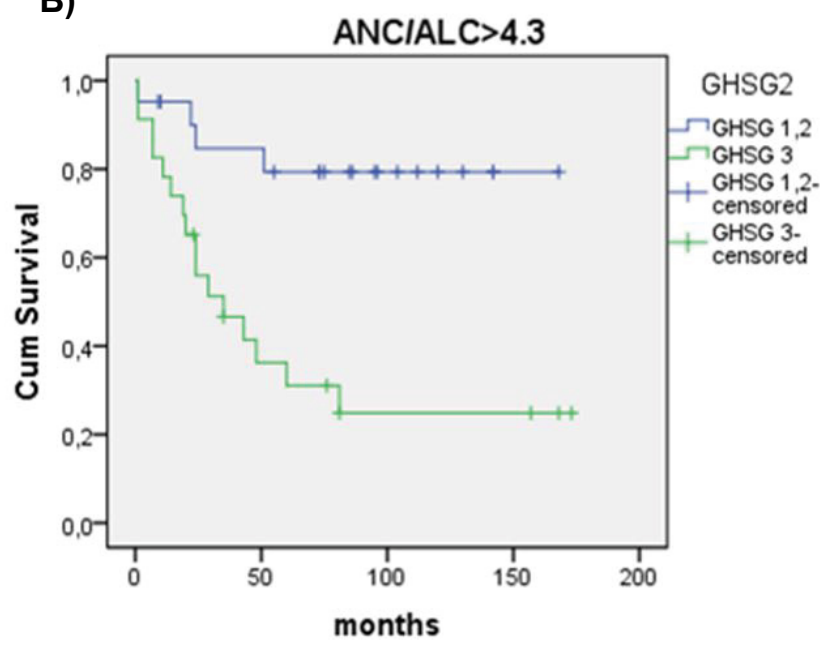

Figure 4: (a,b) Comparison of GHSG (un)favorable early vs. advanced GHCG stage disease in relation to NLR ( $\leq 4.3 \mathrm{vs}$. $\geq 4.3)$.

A)

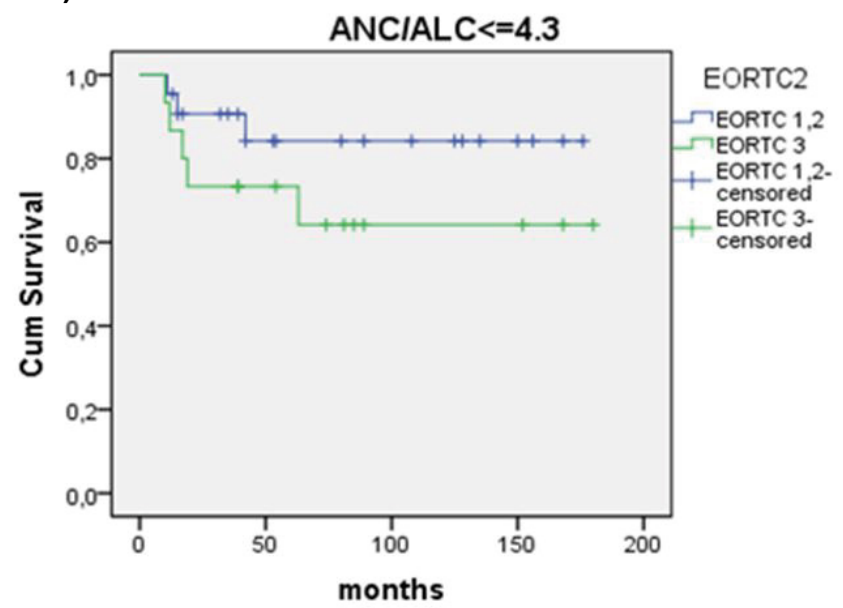

B)

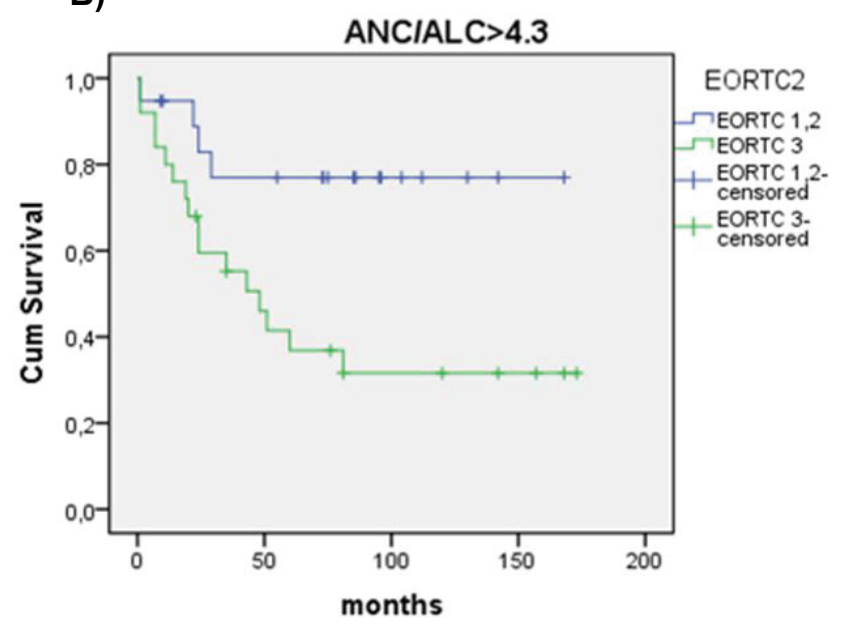

Figure 5: (a,b) Comparison of EORTC (un)favorable early vs. advanced EORTC stage disease in relation to NLR ( $\leq 4.3 v s$. $\geq 4.3$ ).

there is a continuing need for identification of new prognostic factors that may be useful for risk stratification and predicting the response to treatment [15]. To address this issue and better stratify the risk, tests for various prognostic factors have been suggested, including gene expression profiling [16], immunohistochemically analysis of biomarkers, and positron emission tomography [17]. But, not all countries have resources to implement these advanced and sophisticated methods. Current gold standard for risk stratification in $\mathrm{HL}$ word wide is the IPS, but this prognostic score is generated for advanced $\mathrm{HL}$.

In addition, as for any mathematical cutoff value applied to biological phenomenon, one has to be cautions when dealing with "borderline cases". For example, hypothetical patients with $\mathrm{HL}$ and WBC of $14.9 \times 10^{9} / \mathrm{l}$ and lymphocyte count of $0.61 \times 10^{9} / \mathrm{I}$ accounts for zero points in IPS system, regardless of his obviously very high NLR. In addition, NLR should be used for more precise estimation of prognosis in "borderline cases" of HL as well as in clinical stage with carry specific treatment dilemmas, like early (un)favorable stage disease. All these were the reasons why we analyzed NLR in different disease stage according to GHSG and EORTIC staging system. Our results clearly showed that NLR $\geq 4.3$ can distinguish poorer prognosis in early (un)favorable stage in comparison to advanced stage disease.

Pathologically, $\mathrm{HL}$ is characterized by the presence of a small number of diagnostic Reed-Sternberg cells in a background of bystander reactive cells composed of lymphocytes, neutrophils, macrophages, eosinophils, and plasma cells. Lymphopenia is related to adverse survival outcoma [17], as well as tumor-infiltrating lymphocytes and tumor-infiltrating macrophages that constitute tumor microenvironment $[12,18,19]$. Tumor-infiltrating lymphocytes and tumor-infiltrating macrophages are reported to be prognostic factors for survival in patients with $\mathrm{HL}$ [16]. The tumor microenvironment and, in particular, 
Citation: Hajder J, Stanisavljević N, Marisavljević D, et al. (2021) Neutrophil to Lymphocite Ratio Predicts Overall Survival in Newly Diagnosed Hodgkin Lymphoma Patients-Single Centre Experience. Clin Hematol Res 4(1):47-54

the inflammatory response play an important role in cancer development and progression and may be associated with systemic inflammation. NLR can be used as a marker of systemic inflammation since it has long been known that the baseline raised white blood cell count $\left(\geq 15 \times 10^{9} / \mathrm{L}\right)$ and lower lymphocyte count has a prognostic role in classic Hodgkin lymphoma.

The accumulation of neutrophils is related to increased cytokine levels, especially IL-8 $[12,20]$ and it has been suggested that a high number of neutrophils may actually promote tumor growth and metastasis and/or inhibit lymphocyte activity, thereby counteracting the antitumor immune response $[13,21]$. These observations suggest that an imbalance in the ratio of neutrophil to lymphocyte in the peripheral blood of patients with cancer may be related to tumor development. However, the number of studies related to the clinical significance of NLR, especially on the longterm results of hematological malignancies are quite a few [14], when compared to those carried out with solid tumors [22]. Our single center study clearly showed that high NLR is associated with shorter overall survival in patients with newly diagnosed $\mathrm{HL}$. The quick, accessible, inexpensive, easily applicable parameter is strong indicator of shorter survival in patients with advanced disease. However, comprehensive prospective studies in larger cohorts of $\mathrm{HL}$ patients are needed to evaluate prognostic significance of NLR in early stage disease. The authors declare that they have no conflict of interest.

\section{References}

1. Engert A, Younes A (2012) Hodgkin lymphoma: A comprehensive overview. Gallamini A, Hutchings $M$ Functional imaging in Hodgkin lymphoma 107-123.

2. Hasenclever D, Diehl V (1998) A prognostic score for advanced Hodgkin's disease. N Engl J Med 339: 1506-1514.

3. Coussens LM, Werb Z (2002) Inflammation and cancer. Nature 420: $860-867$

4. Cao Y, Shi YX, Chen JO, et al. (2012) Serum C- reactive protein as an important prognostic variable in patients with diffuse large $B$ cell lymphoma. Tumour Biol 33: 1039-1044.

5. Mohri Y, Tanaka K, Ohi M, et al. (2010) Prognostic significance of host- and tumor-related factors in patients with gastric cancer. World J Surg 34: 285-290.

6. Troppan K, Deutsch A, Gerger A, et al. (2014) The derived neutrophil to lymphocyte ratio is an independent prognostic factor in patients with diffuse large B-cell lymphoma. Br J Cancer 110: 369-374.

7. Walsh SR, Cook EJ, Goulder F, et al. (2005) Neutrophil lymphocyte ratio as a prognostic factor in colorectal cancer. J Surg Oncol 91: 181-84.

8. Zhang DS, Wang DS, Wang ZQ, et al. (2012) Comparison of the prognostic values of various inflammation based factors in patients with pancreatic cancer. Med Oncol 29: 3092-3100.
9. Szkandera J, Absenger G, Liegl Atzwanger B, et al. (2013) Elevated preoperative neutrophil/lymphocyte ratio is associated with poor prognosis in soft-tissue sarcoma patients. Br J Cancer 108: 1677-1683.

10. Pichler M, Hutterer GC, Stojakovic T, et al. (2013b) High plasma fibrinogen level represents an independent negative prognostic factor regarding cancer-specific, metastasis-free, as well as overall survival in a European cohort of non-metastatic renal cell carcinoma patients. Br J Cancer 109: 1123-1129.

11. Porrata LF, Ristow K, Habermann T, et al. (2010) Predicting survival for diffuse large $B$-cell lymphoma patients using baseline neutrophil/lymphocyte ratio. Am J Hematol 85: 896-899.

12. Burak UZ (2016) Usefullness of baseline neutrophil to lymphocyte ratio (NLR) in Hematological malignancies. Global Journal of Hematology and Blood Transfusion 3: 10-15.

13. Young Wha Koh, Hyo Jeong Kang, Chansik Park, et al. (2012) Prognostic significance of the ratio of absolute neutrophil count to absolute lymphocyte count in classic Hodgkin lymphoma. Am J Clin Pathol 138: 846-854.

14. Reddy JP, Hernandez M, Gunther JR, et al. (2018) Pre-treatment neutrophil/lymphocyte ratio and platelet/lymphocyte ratio are prognostic of progression in early stage classical Hodgkin lymphoma. Br J Haematol 180: 545-549.

15. Derenzini E, Younes A (2011) Predicting treatment outcome in classical Hodgkin lymphoma: Genomic advances. Genome Med 3: 26 .

16. Stedil C, Lee T, Shah SP, et al. (2010) Tumor- associated macrophages and survival in classic Hodgkin's lymphoma. N Engl J Med 362: 875-885.

17. Mocikova H, Pytilik R, Markova J, et al. (2011) Pre-transplant position tomography in patients with relapsed Hodgkin lymphoma. Leuk Lymphoma 52: 1668-1674.

18. Alvaro Naranjo T, Lejeune M, Salvadó Usach MT, et al. (2005) Tumor-infiltrating cells as a prognostic factor in Hodgkin's lymphoma: A quantitative tissue microarray study in a large retrospective cohort of 267 patients. Leuk Lymphoma 46: 15811591.

19. Schreck S, Friebel D, Buettner M, et al. (2009) Prognostic impact of tumour-infiltrating Th 2 and regulatory T cells in classical Hodgkin lymphoma. Hematol Oncol 27: 31-39.

20. Skinnider BF, Mak TW (2002) The role of cytokines in classical Hodgkin lymphoma. Blood 99: 4283-4297.

21. Rotondo R, Bertolotto M, Barisione G, et al. (2011) Exocytosis of azurophil and arginase 1 containing granules by activated polymorphonuclear neutrophils is required to inhibit $T$ lymphocyte proliferation. J Leukoc Biol 89: 721-727.

22. Marcheselli R, Bari1 A, Tadmor T, et al. (2016) Neutrophillymphocyte ratio at diagnosis is an independent prognostic factor in patients with nodular sclerosis Hodgkin lymphoma: Results of a large multicenter study involving 990 patients. Hematol Oncol 1-6.

DOI: $10.36959 / 831 / 384$

Copyright: (C) 2021 Hajder J, et al. This is an open-access article distributed under the terms of the Creative Commons Attribution License, which permits unrestricted use, distribution, and reproduction in any medium, provided the original author and source are credited. 